\title{
Development of an amphiphilic chitin derivative with lipid/peptide-type side chains having skin protective ability
}

\begin{abstract}
$\mathrm{N}$-(N'-Dodecanoylglycyl)chitin (DGC) as a new type of amphiphilic polymer of lipid/ peptide/polysaccharide conjugate was evaluated its ability to restore an artificially disrupted skin barrier. Cutaneous barrier disruption improved rapidly following application of DGC solutions, suggesting the polymer had a skin-wrapping effect that protected against water flux and restored the basal cell layer to its normal condition. To examine the protective effect of the DGC membrane in further detail, the penetration of chemicals was investigated. DGC suppressed skin barrier disruption as indicated by increased transepidermal water loss (TEWL) resulting from $\mathrm{n}$-hexadecane or oleic acid treatment. Erythema induced by topical application of 2 irritants, methyl nicotinate and hexyl nicotinate, was not observed in DGCpretreated skin. These results indicated that DGC protected the skin from various stimulants and modulated skin condition via its wrapping ability. We propose that DGC is an effective medical agent for improving sensitive skin and keeping skin healthy.
\end{abstract}

Volume 2 Issue 3 - 2015

\author{
Taizo SEKI,' Masaru OHTANI,' Keigo AOI' \\ 'Department of Applied Molecular Biosciences, Nagoya \\ University, Japan \\ ${ }^{2}$ The University of Tokyo, Japan

\begin{abstract}
Correspondence: Keigo AOI, Department of Applied Molecular Biosciences, Graduate School of Bioagricultural Sciences, Nagoya University, Furo-choChikusa-ku, Nagoya, 464860I, Japan, Tel +8I 52789 4I40, Fax +8I 52789 4I40, Email aoi@agr.nagoya-u.ac.jp
\end{abstract}

Received: December 25, 2014 | Published: June 24, 2015

Keywords: chitin/chitosan, polymeric biomaterials, topical application, stratum corneum

Abbreviations: SC, stratum corneum; TEWL, transepidermal water loss; WSC, water-soluble chitin; GlcN, 2-amino-2-deoxy-b-dglucopyranose; GlcNAc, 2-acetoamido-2-deoxy-b-D-glucopyranose; HOPDS, dimethyl-4-hydroxyphenylsulfonium methyl sulphate; DCC, dicyclohexyl carbodiimide; SDS, sodium dodecyl sulphate; MN, methyl nicotinate; HN, hexyl nicotinate, NMR, nuclear magnetic resonance; IR, infrared; SEC, size exclusion chromatography; IL, interleukin; TGF, transforming growth factor; LC, langerhans cell; DC, dendritic cells; KC, keratinocyte; TJ, tight junction; WDEIA, wheat-dependent exercise-induced anaphylaxis

\section{Introduction}

Chitin, aß-(1 $\rightarrow 4)$-linked 2-deoxy-2-acetamido-D-glucopyranosyl polymer, is widely distributed in nature including arthropod shells and fungal cell walls. ${ }^{1}$ Chitin is derived from the Greek word chiton, which was a form of clothing in Ancient Greece. Chitosan is produced by deacetylation of chitin, and glucosamine, one of the most abundant monosaccharides, is produced by the hydrolysis of chitosan. These compounds are already used in medical, functional food, and cosmetic fields. ${ }^{2}$

Chitin, chitosan, and various modifications have been reported to demonstrate unique biofunctional properties such as antitumor activity, immune enhancement, and acceleration of wound healing. ${ }^{3}$ The wound healing properties of chitin and its derivatives have been particularly well characterized. ${ }^{4,5}$ Chitin/chitosan-based dressings such as Beschitin ${ }^{\circledR}$ (Unitika, Japan) and HemCon ${ }^{\circledR}$ (HemCon Medical Technologies Inc. USA) have been found to accelerate wound healing properties and the appearance of healed skin in medical applications. ${ }^{6,7}$ In a randomised controlled trial, an advanced hydrophilic wound dressing containing chitosan was more effective in treating wounds than conservative treatment (gauze) ${ }^{8}$ Recently, a nanometres-thick adhesive bandage sheet called a nanosheet made from chitosan and alginate sodium was developed for surgical use on internal organs. ${ }^{9}$ In addition to an enhanced healing effect, the drug entrapment efficiency of chitin/chitosan allows a wide range of topically pharmaceutical applications to microencapsulate drugs such as hydrogels, films and microspheres. ${ }^{10-15}$

Various modifications of chitin and chitosan have been investigated for their practical applicability and in light of the intractable bulk structure of chitin. ${ }^{16-18}$ Water-soluble chitin (WSC), which is $50 \%$ randomly deacetylated, is known to be soluble in water at room temperature. ${ }^{19}$ WSC has a novel crystal structure that lacks the highly ordered crystalline structure of raw a-chitin formed by hydrogen bonds between hydroxyl groups and amide groups in the repeating unit. ${ }^{20}$ WSC has been reported as a more effective wound-healing accelerator than chitin and chitosan. ${ }^{21}$ Moreover, derivatisation of WSC is one important technique to introduce various biological functions to chitin. We have previously reported a graft copolymerbased WSC that not only demonstrated high solubility in polar solvents but miscibility with common polymers, such as chitin-graftpoly(2-alkyl-2-oxazoline) and chitin-graft-polysarcosine. ${ }^{22-30}$

Since chitin is assumed to have polypeptide side chains linked directly to some of the $\mathrm{C}-2$ amino groups under intact conditions, we have designed chitin derivatives carrying various peptides at the $\mathrm{C}-2$ position with the aim to endow chitin with valuable functions. ${ }^{31}$ In this study, we prepared $N$-( $N^{\prime}$-acetylglycyl) chitin (AGC) and $N-\left(N^{\prime}-\right.$ dodecanoylglycyl)chitin (DGC) with amphiphilic lipoamino acid side groups to achieve higher affinity with the hydrophobic stratum corneum (SC).

Recently, laser and light skin treatments have become popular modalities for the rejuvenation treatment of cosmetic surgery patients. For example, fractional laser resurfacing is a method of skin rejuvenation for use on wrinkles around the eyes and age spots. The 
skin is treated with fractional laser radiation, which is spaced out at a microscopic level as tiny spots, and old skin cells are replaced with new, healthy cells. Chemical peeling uses a chemical solution to remove the outer layers of skin and improve acne. These cosmetic treatments induce skin barrier disruption as a side effect. Furthermore, the number of individuals with atopic skin has been steadily increasing due to drastic changes in the environment. Atopic skin types usually demonstrate dryness, and the barrier function of atopic skin has also been observed as seriously diminished. Deficiency in filaggrin, a skin protein that helps maintain skin moisture and barrier function, is thought to be a major risk factor for individuals with atopic dermatitis. ${ }^{32,33}$ It is thought that it becomes easy for environmental factors such as mites and house dust, perspiration, bacteria or fungi, contact antigens, stress, and food to influence symptoms of atopic dermatitis due to the loss of the skin barrier. The ability of various irritants to penetrate through the skin due to decreasing barrier function worsens the symptoms of skin disease and after-effects of cosmetic medical treatments. Recently, we reported that the dermal irritation of all-trans retinoic acid (tretinoin) could be controlled using hydroxypropyl-b-cyclodextrin (HP- b-CyD) by the clinical test. ${ }^{34,35}$

Cyclodextrins, water-soluble polymers, polymeric micelles and nanospheres made of the biodegradable polymer are used as drug carriers for non-covalent binding of drugs. Therefore, the hypothesis was carried out when the percutaneous absorption of the cutaneous irritant could be controlled by macromolecular drug carriers. We considered the application of amphiphilic chitin derivatives as functional polymers to support the skin barrier. Skin barrier function was assessed by measuring transepidermal water loss (TEWL). ${ }^{36}$ TEWL is a measurement of the quantity of water that passes from inside a body through the skin via evaporation processes. The measurement of TEWL provides an early quantitative determination of damage to the skin-water barrier.

In the present study, we prepared DGC and AGC from WSC by the water-soluble active ester method in water and investigated the beneficial effects of DGC on skin barrier function. The dorsal skin of hairless mice was treated with chemicals, $n$-hexadecane, oleic acid, and $3.0 \mathrm{wt} \%$ aqueous solution of sodium dodecyl sulphate, to remove skin barrier function as estimated by TEWL value. Before or after disruption of skin barrier function, the dorsal skin of hairless mice was also treated with the DGC solution. In addition, the dorsal skin of guinea pigs was treated with DGC solution followed by erythemainducing nicotinates. This study demonstrates that treatments with this novel chitin-based amphiphilic polymer are effective both for preventive and therapeutic skincare in these animal models of sensitive skin.

\section{Materials and methods}

\section{Materials}

We have already described a facile synthesis of chitin derivatives carrying peptide pendant groups by means of the water-soluble active ester method in water (Figure 1). The structures of watersoluble chitin (WSC), $N$-( $N$ '-acetylglycyl)chitin (AGC), and $N$-( $N$ '-dodecanoylglycyl)chitin (DGC) are shown in Figure 2. DGC was synthesised from the water-soluble active ester of $\mathrm{N}$-dodecanoylglycine (Ajinomoto Co. Inc.) and WSC (Marinedew PC$100 \AA$, partially deacetylated chitin, degree of acetylation $(\mathrm{DA})=50 \%$, Ajinomoto Co. Inc.) according to a previous method (Figure 3). The water-soluble active ester of $N$-dodecanoylglycine was synthesised from $N$-dodecanoylglycine and dimethyl-4-hydroxyphenylsulfonium methyl sulphate (HOPDS; Sanshin Chemical Industrial Co. Ltd.) with condensing agent dicyclohexylcarbodiimide (DCC; Wako Pure Chemical Industries Ltd.). DGC was refined repeatedly by reprecipitation. AGC was synthesised from $N$-acetylglycine (Tokyo Kasei Kogyo Co. Ltd.) and the same WSC in a similar manner. Viscous solutions of DGC and AGC were prepared with $0.2 \mathrm{wt} \%$ lactic acid solutions by mixing overnight at $0.6 \mathrm{wt} \%$ or $1.0 \mathrm{wt} \%$ concentration (pH 6.0). For comparison, WSC aqueous solution with lactic acid and vehicle solution of lactic acid were used.

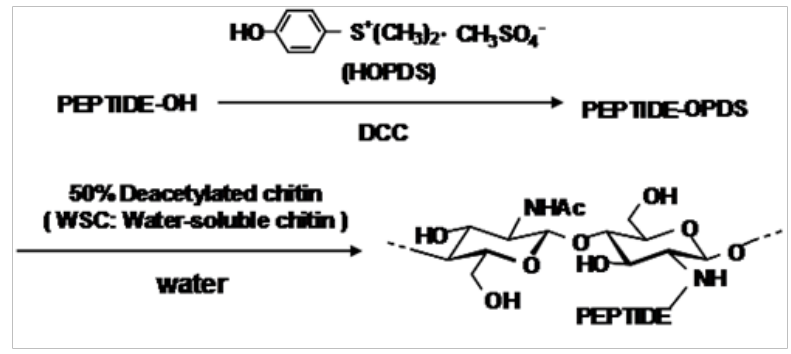

Figure I N=57; Epidemiological distribution of the pathological fractures, traumatic fractures, and nonunion.
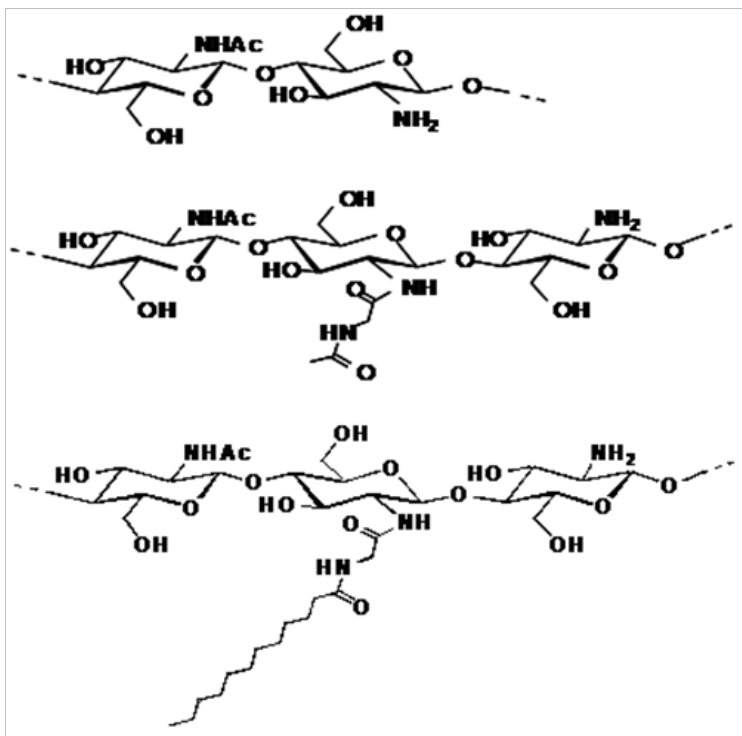

Figure 2 Structures of water-soluble chitin (WSC), Acetylglycylchitin (AGC) and Dodecylglycylchitin (DGC), sequentially.

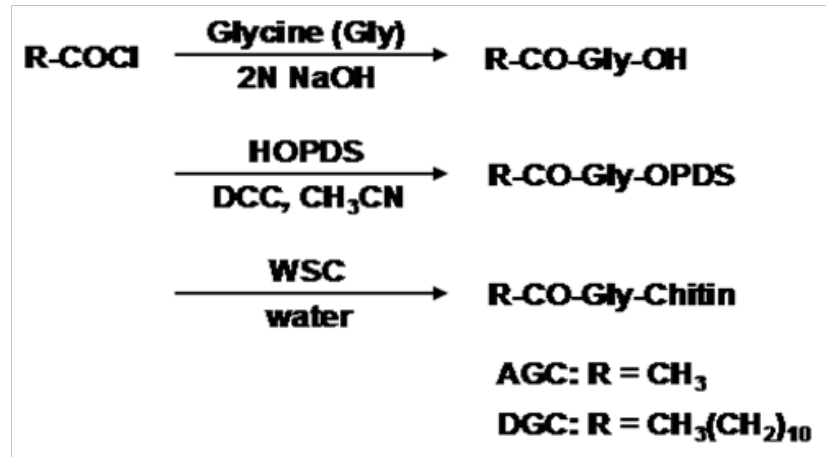

Figure 3 Synthesis of Dodecylglycylchitin (DGC) and Acetylglycylchitin (AGC) from water-soluble chitin (WSC).

HOPDS, dimethyl-4-hydroxyphenylsulfonium methyl sulphate; DCC, dicyclohexylcarbodiimide 


\section{Structure determination of chitin derivatives}

The structure of chitin derivatives was identified using NMR and IR spectroscopy. ${ }^{1} \mathrm{H}$ and $13 \mathrm{C}$ NMR measurements were performed with a Bruker ARX 400 spectrometer at 400 and $100 \mathrm{MHz}$, respectively. IR spectra were recorded with a JASCO FT/IR-610 spectrometer. Size exclusion chromatography (SEC) was conducted with a JASCO Model PU-980 high performance liquid chromatography apparatus (column, Shodex B-805 $\rightarrow$ B-804; eluent, $0.5 \mathrm{M}$ acetic acid $+0.5 \mathrm{M}$ sodium acetate; $27^{\circ} \mathrm{C}$; flow rate, $1.0 \mathrm{~mL} / \mathrm{min}$ ).

\section{Measurement of transepidermal water loss (TEWL)}

The improvement of skin barrier function was estimated by the measurement of TEWL using a Tewameter ${ }^{\circledR}$ CR200 $(C+K$ electronic, Cologne, Germany). The measurement principle is based on the estimation of the vapour pressure gradient in the zone of diffusion immediately adjacent to the surface of the skin (Figure 4). The TEWL value increases with cutaneous barrier disruption and can quantitatively express the degree of barrier function improvement Hairless mice and guinea pigs were used in TEWL experiments. All animal studies were carried out under the guidelines for animal experiments of Nagoya University (Nagoya, Japan) and Law No.105 and Notification No.6 of the Prime minister's Office of Japan.

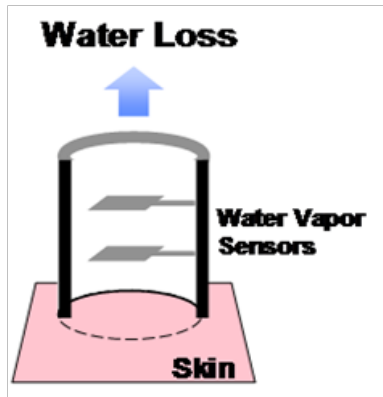

$$
\frac{d m}{d t}=\mathbf{D}-\mathbf{A} \frac{d \sigma}{d t}
$$

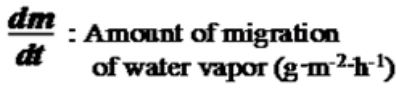

D : Coefficient of diffusion of water vapor

A : Volume

\section{$\frac{d \sigma}{d l}$ : Change in density of water vapor per unit length}

Figure 4 The measurement principle is based on the estimation of the vapour pressure gradient in the zone of diffusion immediately adjacent to the surface of the skin. The transepidermal water loss (TEWL) value is increased by cutaneous barrier disruption and can quantitatively express the degree of improvement in barrier function.

\section{Creation of skin barrier dysfunction and recovery effect of chitin derivatives}

Hairless mice ( $\mathrm{n}=15$, female, HR-1, 6weeks old) were first divided into 3 groups of 5 and maintained under conventional conditions. Three days later, $\mathrm{n}$-hexadecane $(40 \mathrm{~mL})$ was applied to the dorsal skin of each mouse twice daily. In approximately 1week, cutaneous barrier disruption was confirmed by TEWL values consistently exceeding $20 \mathrm{~g} \cdot \mathrm{m}^{-2}<\bullet \mathbf{h}>-1$. This skin barrier disruption model is non-invasive, does not involve an external wound, and the pain to the animal may be very low. Samples of DGC, AGC, and WSC aqueous solutions (1.0 $\mathrm{wt} \%, 100 \mathrm{~mL}$ ) were then applied to this artificially barrier-disrupted dorsal skin twice daily. The degree of improvement in skin barrier disruption was assessed by TEWL on the dorsal skin of 5 specified mice in each group after sufficient air drying (Figure 5). After 55hours, the dorsal skin of the one remaining hairless mouse of each group was extracted to observe histological findings. The data from this individual was not used for statistical analysis.

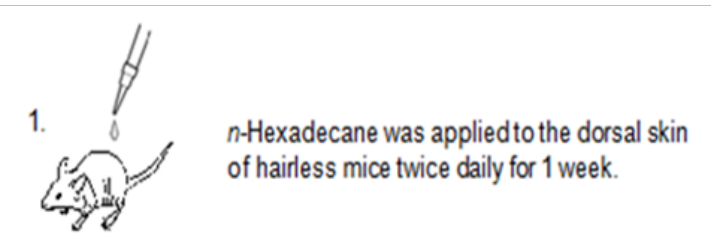

2. Gr

Cutaneous barrier disruption was confirmed

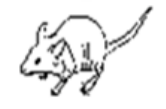
by TEWL values exceeding $20 \mathrm{~g} \mathrm{~m}^{2} \cdot \mathrm{h}^{-1}$

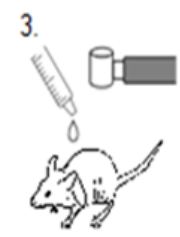

Samples (1.0 wt \% aqueous solution, $100 \mu \mathrm{L}$ ) were applied twice daily on dorsal skin. The degree of improvement was estimated by TEWL.

Figure 5 Experimental protocols for investigating recovery of skin barrier function. Acetylglycylchitin (AGC), Dodecylglycylchitin (DGC) and watersoluble chitin (WSC) were dissolved in water at the appropriate concentrations with roughly equimolecular amounts of lactic acid to residual amino groups.

\section{Preventive effect from chemical disturbance of skin barrier function}

Hairless mice ( $\mathrm{n}=15$, female, HR-1, 6week old) were divided into 3 groups of 5 and maintained under conventional conditions. Based on the results of the first experiment, only DGC was used in this study and compared with WSC and vehicle solution. Because $1.0 \mathrm{wt} \%$ chitin solution seemed too viscous for use as a preventive agent, test samples were diluted slightly with water to $0.6 \mathrm{wt} \%$. For 3days, test sample solutions $(0.6 \mathrm{wt} \%, 100 \mathrm{~mL})$ of DGC, WSC, or vehicle were applied to the dorsal skin of hairless mice twice daily. After the samples were applied, chemicals n-hexadecane $(40 \mathrm{~mL})$, oleic acid $(40 \mathrm{~mL})$, or 3.0 wt $\%$ sodium dodecyl sulphate (SDS) solution were applied to the skin once. The preventive effect on maintaining skin barrier function was evaluated by assessing changes in TEWL (Figure 6). The dorsal skin of each hairless mouse was then extracted to observe histological changes.

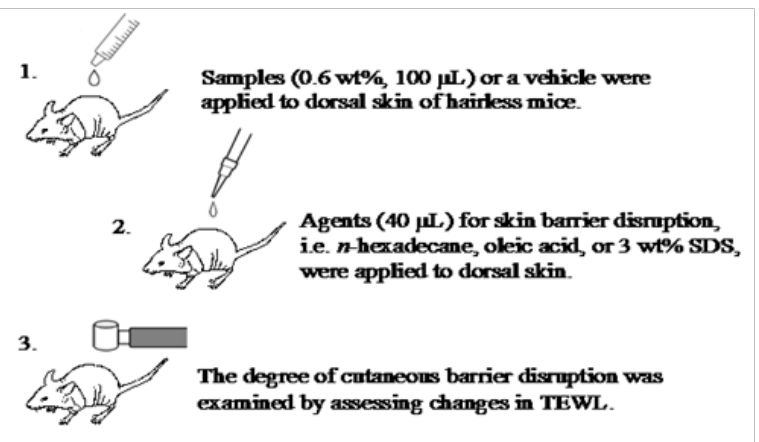

Figure 6 Experimental protocols for investigating the preventive effect from chemical skin barrier disturbance. Dodecylglycylchitin (DGC) and water-soluble chitin (WSC) were dissolved in water at the appropriate concentrations with roughly equimolecular amounts of lactic acid to residual amino groups. Vehicle (lactic acid aqueous solution neutralized at $\mathrm{pH}$ 6) was used as a control. 


\section{Reduction of nicotinate-induced erythema}

We used methyl nicotinate $(\mathrm{MN})$ in water and hexyl nicotinate $(\mathrm{HN})$ in olive oil as irritants, and applied them to the dorsal skin of guinea pigs $(\mathrm{n}=5$, female, SPF, 5weeks old) as a model of experimentally induced erythema. After preoperative shaving and washing of the dorsal skin with tepid water, irritants were close-patched onto the dorsal skin with Torii-BanÒ (Torii Pharmaceutical Co. Ltd.) for 10min. Erythema was scored visually at $5 \mathrm{~min}$ after removal of the irritant (Figure 7), with 0 $=$ not detected, $1=$ questionable, and $2=$ marked erythema.

1.

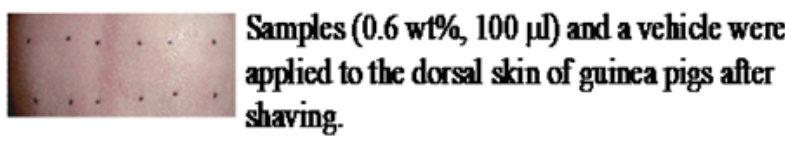

2.

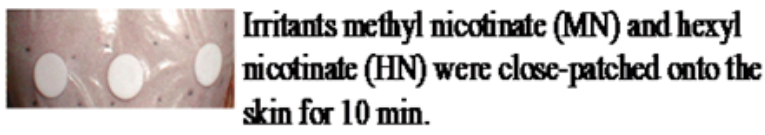

3.

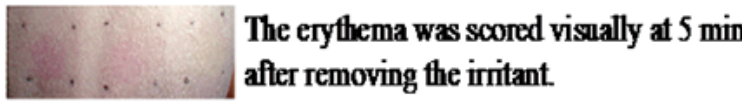

Figure 7 Experimental protocols for reduction of erythema induced by nicotinates. Dodecylglycylchitin (DGC) and water-soluble chitin (WSC) were dissolved in water at the appropriate concentrations with roughly equimolecular amounts of lactic acid to residual amino groups. Vehicle (lactic acid aqueous solution neutralized at $\mathrm{pH}$ 6) was used as a control. Visual scores were classified as follows: $0=$ not detected, $\mathrm{I}=$ questionable, $2=$ marked erythema.

To obtain the threshold value of erythema on each individual, MN in water $(10 \mathrm{~mL})$ and $\mathrm{HN}$ in olive oil $(30 \mathrm{~mL})$ were close-patched onto the dorsal skin of guinea pigs at concentrations ranging from 0.005 $\mathrm{wt} \%$ to $0.3 \mathrm{wt} \%$. The threshold values of erythema were approximately $0.03 \mathrm{wt} \%$ for $\mathrm{MN}$ and $0.05 \mathrm{wt} \%$ for $\mathrm{HN}$. Samples $(0.6 \mathrm{wt} \%, 100 \mathrm{~mL})$ of DGC, WSC, or vehicle solution were applied to a $1.5 \mathrm{~cm}$ square of the dorsal skin of guinea pigs. After the treated areas were air-dried, $\mathrm{MN}$ in water $(10 \mathrm{~mL})$ and $\mathrm{HN}$ in olive oil $(30 \mathrm{~mL})$ at the concentration of each individual's threshold values to induce erythema were closepatched for $10 \mathrm{~min}$. After removing the patch from the skin and waiting $5 \mathrm{~min}$, erythema was visually scored as described above.

\section{Histological examination}

Skin samples were treated immediately after excision under anaesthesia. Specimens were fixed in $10 \%$ buffered formalin for $24 \mathrm{~h}$ and embedded in paraffin following dehydration. Sections were cut with a microtome and stained with haematoxylin-eosin according to standard practice.

\section{Statistical analysis}

In the investigation of the degree of improvement in skin barrier function caused by DGC, AGC, and WSC, homogeneity of variances and mean TEWL values in the 3 groups of skin barrier disruption models were confirmed by Levene's test and one-way ANOVA, respectively. Differences between treatment means were then tested by a two-way ANOVA, followed by Tukey's multiple-comparison post-hoc test. In the evaluation of the preventive effect of DGC for chemical disturbance of skin barrier function, homogeneity of variances and mean TEWL values after assignment of hairless mice to the 3 treatment groups (DGC, WSC, or vehicle solution) were confirmed by Levene's test and one-way ANOVA, respectively. Differences between pre-treatment means before and after chemical treatment were determined using Tukey's test. In the investigation of nicotinate-induced erythema reduction on the dorsal skin of guinea pigs, the statistical significance of differences across the 3 groups was calculated using the Steel-Dwass test for visual erythema scores.

\section{Results and discussion}

\section{Preparation and characterization of DGC and AGC}

WSC is known to form a gel in water. The WSC aqueous solution was prepared by pouring WSC powder into crushed ice and stirring with a muddler. WSC then gradually dissolved in the water as it reached room temperature. Without the protection of the hydroxyl groups of chitin, the amino groups of WSC were selectively acylated by the water-soluble active ester method in a homogeneous system in water. The obtained products demonstrated water solubility and could be reprecipitated from a reactive solution poured and washed into an organic solvent such as acetone. The structures of WSC, AGC, and DGC were determined by ${ }^{1} \mathrm{H} \mathrm{NMR}$ in $\mathrm{D}_{2} \mathrm{O} / \mathrm{CD}_{3} \mathrm{CO}_{2} \mathrm{D}(95: 5, \mathrm{v} / \mathrm{v})$ at $60^{\circ} \mathrm{C}$. The ${ }^{1} \mathrm{H}$ NMR signals of chitins were broad due to the polymer. The degree of $N$-acetylation (DA) of the initial WSC was determined as 0.5 from the integral intensities of the $\mathrm{H}^{-1}$ of the 2-amino-2deoxy- $\beta$-D-glucopyranose $(\mathrm{GlcN})$ unit at $4.8 \mathrm{ppm}$ and the $\mathrm{H}^{-1}$ of the 2 -acetoamido-2-deoxy- $\beta$-D-glucopyranose (GlcNAc) unit at 4.6ppm.

In the case of AGC and DGC, the NMR signals of $\mathrm{H}-1$ protons of the $N$-acyl GlcN units, i.e. signals of the H-1 proton of $N-(N$ 'acetylglycyl)GlcN, of $N$-( $N^{\prime}$-dodecanoylglycyl)GlcN, and of GlcNAc, overlap at 4.6ppm. The $\mathrm{H}^{-1}$ signal of GlcN appears at 4.8ppm. The degree of substitution (DS) of AGC and DGC was then calculated using the intensities of the signals at 4.6 and $4.8 \mathrm{ppm}$. Because of the difference in longitudinal relaxation time $\mathrm{T} 1$ between chitin main chain and pendant side chain, the intensities of the pendant side chain signals seem easy to overestimate. ${ }^{37}$ Thus, only $\mathrm{H}^{-1}$ protons on the chitin main chain were used for determination of DS. In a previous study, the DGC degree of substitution (DS) was determined by ${ }^{1} \mathrm{H}$ NMR spectra to approach $30 \%$ at best. ${ }^{31}$ The DS of WSC derivatives synthesized by the water-soluble active ester method could be roughly controlled by the amount of the active ester, reaction temperature, and reaction times. In the present study, the DS of DGC and AGC prepared from the same WSC were approximately 0.1 (Table 1). The DS of DGC and AGC was defined as the ratio of the amount of dodecanoyl groups and amino groups of initial WSC, that is, $[\mathrm{DG}] /\left[\mathrm{NH}_{2}\right] 0$. Thus, these chitins consisted of $50 \%$ GlcNAc units, $45 \%$ GlcN units, and $5 \% \mathrm{~N}$-acylglycyl GlnN units. Yields from both structures were quantitative.

The apparent number-average molecular weight $\left(\mathrm{M}_{\mathrm{n}}\right)$ and polydispersity $\left(\mathrm{M}_{\mathrm{w}} / \mathrm{M}_{\mathrm{n}}\right)$ of chitins were estimated by SEC. Although DGC and AGC were prepared from same WSC, $M_{n}$ and $M_{w} / M_{n}$ of DGC and AGC may be slightly smaller than the initial WSC. Degradation did not appear to occur under such mild conditions, because in general commercial chitin and WSC are prepared under more severe condition involving strong alkaline treatment and high temperature. $M_{n}$ values decreased from WSC $\left(M_{n}=3.9 \times 10^{5}\right)$ to AGC $\left(M_{n}=3.0 \times 10^{5}\right)$ and DGC $\left(M_{n}=1.5 \times 10^{5}\right)$, and the width of $M_{w} /$ $M_{n}$ values narrowed in the same order (WSC, AGC, DGC). This finding indicates that the hydrodynamic volume of DGC and AGC determined using pullulan as a standard was reduced compared to WSC. AGC and DGC contain amide bonds from pendant glycine units that form intramolecular interactions by hydrogen bonding. In 
addition, DGC contains pendant dodecanoyl units that seem to form intramolecular micelles by hydrophobic interaction. Glycine spacers between hydrophobic pendant units and hydrophilic main chain may be effective to form intramolecular interactions such as unimolecular micelles. When intermolecular interactions are superior, a gel network may be formed. DGC and AGC were dissolved in water for testing samples at appropriate concentrations with a roughly equimolecular amount of lactic acid $(0.2 \mathrm{wt} \%)$ relative to residual amino groups. WSC dissolved in the same manner and a vehicle (LA aqueous solution neutralised at $\mathrm{pH}$ 6) were used as controls in the experiments.

Table I Synthesis of $\mathrm{AGC}^{\mathrm{a}}$ and $\mathrm{DGC}^{\mathrm{b}}$ from WSC${ }^{\mathrm{c}}$ with water-soluble active ester in water

\begin{tabular}{|c|c|c|c|c|c|c|}
\hline & Active esters & Temp ${ }^{\circ} \mathrm{C}$ & Yield \% & $D^{d}$ & $M n^{\mathrm{e} \times 10-5}$ & $M w / M n e$ \\
\hline WSC $^{c}$ & - & - & - & - & 3.9 & 5.2 \\
\hline $\mathrm{AGC}^{\mathrm{a}}$ & $\mathrm{CH}_{3} \mathrm{CO}$-Gly-OPDS & 27 & 99 & 0.1 & 3 & 4.2 \\
\hline $\mathrm{DGC}^{\mathrm{b}}$ & $\mathrm{CH}_{3}\left(\mathrm{CH}_{2}\right)_{10}$ CO-Gly-OPDS ${ }^{f}$ & 27 & 98 & 0.1 & 1.5 & 3.5 \\
\hline
\end{tabular}

a Acetylglycylchitin; b Dodecylglycylchitin; cWater-soluble chitin (DA, degree of N-acetylation, 0.5 ); $d$ Molar ratio of peptide unit to GlcN unit of the initial chitin, by IH NMR; e By SEC in $0.5 \mathrm{M}$ acetic acid + 0.5 M sodium acetate (pullulan standard)

\section{Effect of DGC to accelerate recovery from skin barrier dysfunction}

Sensitive skin has been reported to demonstrate much higher TEWL than normal skin, indicating disturbance of the stratum corneum (SC) barrier function of sensitive skin. ${ }^{38}$ To evaluate the efficacy of DGC on sensitive skin, the recuperative effect of DGC against skin barrier disruption with $n$-hexadecane treated was estimated. Some hydrocarbons are known to cause skin irritation after repeated application. ${ }^{39}$ The TEWL values on the dorsal skin of normal hairless mice were less than $10 \mathrm{~g} \cdot \mathrm{m}^{-2} \bullet \mathrm{h}^{-1}$. After continued treatment with $n$-hexadecane for 1 week, TEWL values on the dorsal skin of hairless mice came to exceed $20 \mathrm{~g} \cdot \mathrm{m}^{-2} \cdot \mathrm{h}^{-1}$ constantly due to cutaneous barrier disruption.

Hairless mice ( $\mathrm{n}=15)$ were divided into 3 groups. To examine their suitability for improving skin disruption, 3 materials, DGC, AGC, and WSC, were tested. The homogeneity of variance of the TEWL values of the remaining hairless mice with skin barrier disruption was confirmed by Levene's test ( $P=0.20$, Table 2$)$. When DGC solutions were applied to barrier-disrupted SC, the TEWL value was effectively suppressed within $24 \mathrm{~h}$. Furthermore, TEWL reached normal levels at $55 \mathrm{~h}$ after DGC application. Tukey's all-pair wise-comparison test was used to identify differences between WSC, AGC, and DGC results after $76 \mathrm{~h}$. TEWL values significantly decreased after DGC treatment compared with WSC and AGC (WSC vs. DGC: $P=0.01$. AGC vs. DGC: $P=0.03$ ), suggesting improved to skin condition. Because TEWL values were observed to continue rising above 20 $\mathrm{g} \cdot \mathrm{m}^{-2} \cdot \mathrm{h}^{-1}$ for some time after $n$-hexadecane treatment stopped (data not shown), WSC and AGC treatment suppressed this moderate rise in TEWL. This finding indicates that WSC and AGC also have an effect on accelerating recovery from skin barrier dysfunction. The histology of skin barrier disruption by $n$-hexadecane treated with DGC or WSC for more than $55 \mathrm{~h}$ is shown in Figure 8. DGC-treated skin shows no abnormal dermal or epidermal thickness due to $n$-hexadecane. However, WSC alone did not induce any improvement in histology. These results indicate that the lipoamino acid side units of DGC play an important role in the repair of skin barrier disruption.

Table 2 Changes in Transepidermal water loss (TEWL) $(\mathrm{g} \cdot \mathrm{m}-2 \cdot \mathrm{h}-\mathrm{I})$ with treatment of test samples on the dorsal skin of hairless mice after skin barrier disruption by $\mathrm{n}$-hexadecane treatment.

\begin{tabular}{|c|c|c|c|c|c|}
\hline & $\begin{array}{l}\text { Groups: sample } \\
\text { n }\end{array}$ & $\begin{array}{l}\text { I:WSCa } \\
4\end{array}$ & $\begin{array}{l}\text { II:AGC }{ }^{b} \\
4\end{array}$ & $\begin{array}{l}\text { III: DGC } \\
4\end{array}$ & \\
\hline & Time, $\mathbf{h}$ & & TEWL $L^{d}, \mathbf{g} \cdot \mathbf{m}^{-2} \cdot \mathbf{h}^{-1}$ & & $P$ value ${ }^{e}$ \\
\hline Skin barrier disruption & 0 & $23.1 \pm 7.0$ & $24.0 \pm 2.7$ & $21.6 \pm 3.2$ & 0.2 \\
\hline \multirow[t]{4}{*}{ Treatment of samples } & 6 & $22.9 \pm 7.0$ & $20.3 \pm 4.2$ & $18.6 \pm 2.8$ & - \\
\hline & 24 & $18.7 \pm 4.0$ & $19.3 \pm 7.8$ & $15.0 \pm 2.8$ & - \\
\hline & 55 & $15.7 \pm 2.6$ & $16.2 \pm 3.3$ & $9.4 \pm 3.2$ & - \\
\hline & 76 & $11.4 \pm 3.0$ & $9.7 \pm 0.9$ & $7.8 \pm 0.6$ & - \\
\hline \multirow[t]{3}{*}{$P$ value $^{f}$} & IVs. II & & & 0.94 & - \\
\hline & IVs. III & & & $0.01 * *$ & - \\
\hline & IIVs. III & & & $0.03 *$ & - \\
\hline
\end{tabular}

a water-soluble chitin; b acetylglycylchitin; $c$ dodecylglycylchitin; $d$ Mean $\pm S D ; e$ tested by one-way ANOVA followed by Levene's test; fTested by two-way ANOVA followed by Tukey's post hoc tests, * $, P<0.05, * *, P<0.01$ 
The lipoamino acid side units of DGC were found to accelerate the capacity to improve skin roughness inherent to chitin. Although chitin has been reported to demonstrate mild immune-enhancing properties, the mechanisms underlying this effect are still unclear. Improved barrier function and decreased epidermal thickness may result from the modulation of water flux and water retention into the SC due to the wrapping ability of DGC as the hydrophilic polymer. The molecular weight of the chitin ( $>105$, according to SEC) is clearly too high to penetrate the SC. Thus, we hypothesised that its membrane inhibited environmental irritants from permeating through the skin. Therefore, subsequent experiments examined the possible effect of DGC to directly protect the skin barrier from irritants.

\section{Preventive effect from chemicals disturbance of skin barrier function}

The prophylactic effect of DGC and WSC against skin roughness caused by disturbing the skin barrier to enhance skin permeability was investigated. Generally speaking, $n$-hexadecane, oleic acid and SDS are accepted skin barrier-disturbing agents known to cause irritation..$^{40}$ After pretreatment with DGC or WSC, each irritant was applied to hairless mouse skin (Tables 3-5). Because the purpose of this experiment was to compare protective skincare efficacy between DGC and WSC, a lower density $(0.6 \mathrm{wt} \%)$ of DGC and WSC was used compared with the above-mentioned test concentration $(1.0 \mathrm{wt} \%)$ aimed at curative skincare.

Table 3 Changes in TEWL ${ }^{a}\left(g \cdot m^{-2} \cdot h^{-1}\right)$ induced by $n$-hexadecane treatment of dorsal skin of hairless mice after pretreatment with the test samples

\begin{tabular}{|c|c|c|c|c|c|c|c|}
\hline Time & & Initial & & & After & & \\
\hline & Groups & I: & II: & III: & I: & II: & III: \\
\hline & Sample & Vehicle & $W_{S C}^{b}$ & $D_{G C}{ }^{c}$ & Vehicle & $W_{S C}^{b}$ & $\mathrm{DGC}^{\mathrm{c}}$ \\
\hline & $n$ & 5 & 5 & 5 & 5 & 5 & 5 \\
\hline \multirow{4}{*}{ TEWL $^{\mathrm{a}}$} & Mean & 8 & 8.8 & 7.6 & 14.3 & 13.3 & 9.3 \\
\hline & SD & 2.3 & 2.1 & 1.5 & 2.6 & 1.7 & 1.4 \\
\hline & Levene's test & & & 0.88 & & & - \\
\hline & One-way ANOVA & & & 0.62 & & & - \\
\hline \multirow[t]{3}{*}{$P$ value $^{d}$} & Tukey's test & IVs. II & & - & & & 0.69 \\
\hline & & IVs. III & & - & & & $0.005^{*}$ \\
\hline & & IIVs. III & & - & & & $0.02 *$ \\
\hline
\end{tabular}

a Transepidermal water loss; b Water-soluble chitin; c Dodecylglycylchitin; d*, P<0.05

Table 4 Changes in TEWLa $\left(g \bullet \mathrm{m}^{-2} \cdot \mathrm{h}^{-1}\right)$ induced by oleic acid treatment of dorsal skin of hairless mice after pretreatment with the test samples

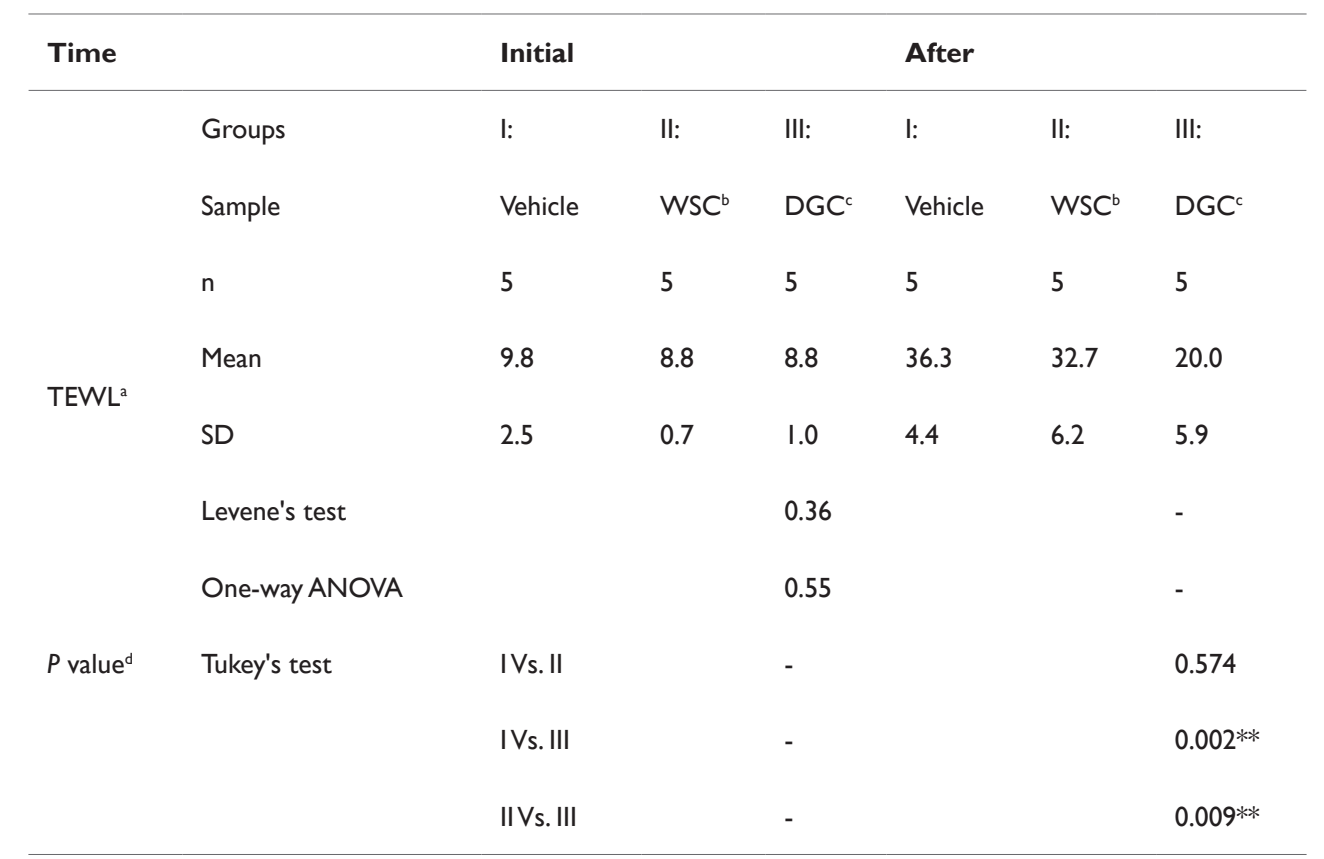

a Transepidermal water loss; b Water-soluble chitin; c Dodecylglycylchitin; $\mathrm{d}$ **, $\mathrm{P}<0.0 \mathrm{I}$ 


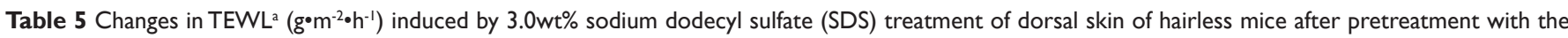
test samples

\begin{tabular}{|c|c|c|c|c|c|c|c|}
\hline Time & & Initial & & & After & & \\
\hline \multirow{5}{*}{$\mathrm{TEWL}^{\mathrm{a}}$} & Groups & I: & II: & III: & I: & II: & III: \\
\hline & Sample & Vehicle & WSC $^{b}$ & $\mathrm{DGC}^{\mathrm{c}}$ & Vehicle & WSC $^{b}$ & $\mathrm{DGC}^{\mathrm{c}}$ \\
\hline & $\mathrm{n}$ & 5 & 5 & 5 & 5 & 5 & 5 \\
\hline & Mean & 6.9 & 6.9 & 6.9 & 19.3 & 16.5 & 18.4 \\
\hline & SD & 1.3 & 0.9 & I.I & I.I & 4.3 & 3.8 \\
\hline \multirow{5}{*}{$P$ value $^{d}$} & Levene's test & & & 0.66 & & & - \\
\hline & One-way ANOVA & & & 0.999 & & & - \\
\hline & Tukey's test & IVs. II & & - & & & $0.4 \mathrm{I}$ \\
\hline & & IVs. III & & - & & & 0.91 \\
\hline & & IIVs. III & & - & & & 0.63 \\
\hline
\end{tabular}

a Transepidermal water loss; b Water-soluble chitin; c Dodecylglycylchitin

a) DGC

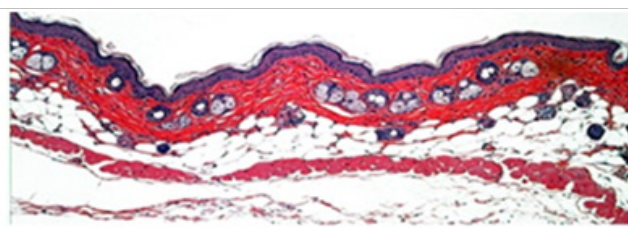

b) WSC

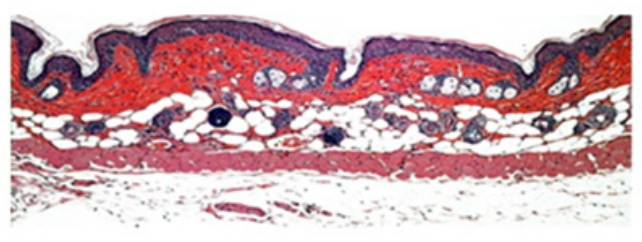

Figure 8 Histological findings from the dorsal skin of hairless mice treated with solutions of dodecylglycylchitin (DGC) (A) or water-soluble chitin (WSC) (B) more than $55 \mathrm{~h}$ after skin barrier disruption by treatment with $\mathrm{n}$-hexadecane (haematoxylin-eosin stain, $\times 100$ ).

After dividing the hairless mice $(\mathrm{n}=15)$ into 3 groups, the homogeneity of variance of the TEWL values was confirmed by Levene's test ( $n$-hexadecane, $P=0.88$; oleic acid, $P=0.36 ; 3.0 \mathrm{wt} \%$ SDS aq. $P$ 0.66). The increase of TEWL values on the dorsal skin of hairless mice induced by $n$-hexadecane treatment was significantly suppressed as a result of DGC pretreatment (vehicle vs. DGC, $P=0.05$; WSC vs. DGC, $P=0.02$ ). The increase of TEWL induced by oleic acid treatment was also significantly depressed by DGC pretreatment (vehicle vs. DGC, $P=0.002$; WSC vs. DGC, $P=0.009$ ). The improvement in TEWL was significantly more pronounced in the DGC-pretreated group than in the WSC or vehicle groups. Using SDS aqueous solution as irritant, no difference was observed between DGC, WSC, and vehicle pretreatments (vehicle vs. DGC, $P=0.91$; WSC vs. DGC, $P=0.63$ ). These data demonstrate that DGC has an excellent prophylactic effect against skin roughness induced by hydrophobic agents that promote skin permeability, such as $n$-hexadecane and oleic acid. For $n$-hexadecane in particular (Table 3), the DGC membrane kept TEWL values normal at $10 \mathrm{~g} \cdot \mathrm{m}^{-2} \cdot \mathrm{h}^{-1}$ or less. However, the prophylactic effect of DGC was not observed for $3.0 \mathrm{wt} \%$ SDS aqueous solution. The histology of skin barrier disruption by $n$-hexadecane with DGC and WSC pretreatment is shown in Figure 9. Skin pretreated with vehicle solutions demonstrated thickening in the dermal and epidermal layers due to $n$-hexadecane. With DGC pretreatment, skin appears to remain normal. Because SDS is a strong surface-active agent, the amphiphilic side units of DGC were solubilised and did not function as barrier in that treatment condition. Although it remains unclear whether DGC inhibited surrounding irritants from penetration through the skin and/ or protected skin barrier function against disturbance by $n$-hexadecane or oleic acid, these results indicated that DGC application prevented the skin barrier dysfunction causative of irritation.

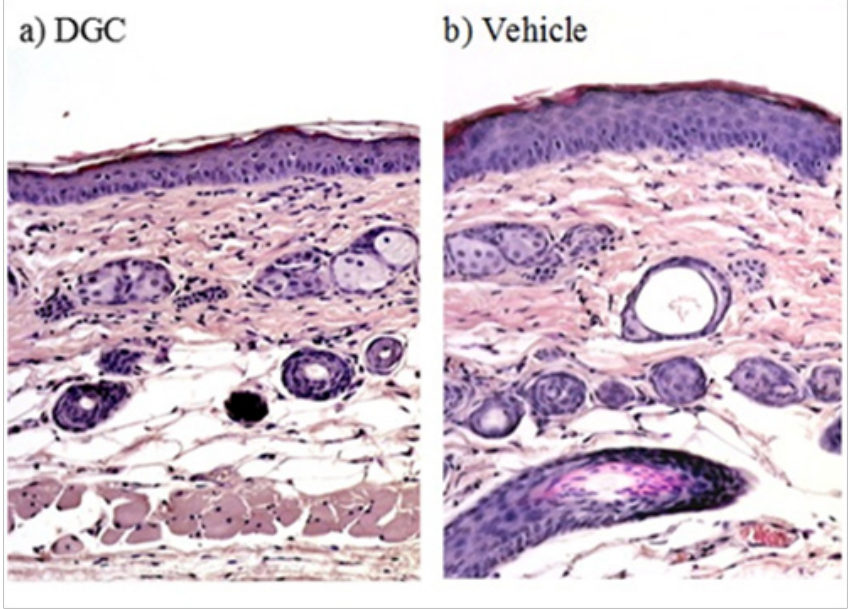

Figure 9 Histological findings from the dorsal skin of hairless mice treated with $n$-hexadecane after pretreatment with dodecylglycylchitin (DGC) (A) or vehicle control (B). No epidermal thickening was observed in skin treated with n-hexadecane after DGC pretreatment (haematoxylin-eosin stain, $\times 100$ ).

\section{Reduction of erythema induced by nicotinates}

Nicotine derivatives including hydrophilic $\mathrm{MN}$ and lipophilic $\mathrm{HN}$ are well known to possess a vasodilatative effect and induce transient erythema on human skin..$^{41}$ The observation of erythema on guinea pig skin by these compounds has also been reported. ${ }^{42}$ Thus, the inhibitory effects of DGC on skin penetration of nicotinates were examined using erythema as an indication. To evaluate the skin penetration of nicotinates by semiquantitative visual scoring, we confirmed this phenomenon on guinea pig skin (female, SPF, 5weeks old), and then determined the individualminimum concentrations of both $\mathrm{MN}$ in 
water and $\mathrm{HN}$ in olive oil by which erythema was induced prior to experiments. $\mathrm{MN}$ was used as a representative water-soluble irritant, while $\mathrm{HN}$ was adopted as an oil-soluble irritant.

As shown in Table 6, DGC significantly inhibited skin from developing symptoms of MN-induced erythema compared to WSC and vehicle (vehicle vs. DGC, $P=0.047$; WSC vs. DGC, $P=0.047$ ). $\mathrm{HN}$-induced erythema on the dorsal skin of guinea pig was also significantly suppressed by treatment with DGC compared to vehicle (vehicle vs. DGC, $P=0.037$; Table 6). These experimental results indicated that DGC suppressed the penetration of both hydrophilic $\mathrm{MN}$ and lipophilic HN irritants into the skin. Because sugar units forming the chitin backbone possess higher polarity, it is reasonable that DGC prevents skin from the penetration of lipophilic substances. Lipophilic side groups may be responsible for the blocking effect against hydrophilic irritants.

To create a moist environment for rapid wound healing, many biomaterials composed of chitin/chitosan and other hydrophilic polymers have been developed as functional wound dressings. WSC was reported to be a more effective wound-healing accelerator than chitin or chitosan. ${ }^{21}$ In this study, we investigated whether DGC prepared from WSC was a more effective accelerator of healing the skin barrier disruption than WSC. The rate of skin barrier recovery increased in the following order: WSC $<$ AGC $<$ DGC. DGC containing amphiphilic side groups may form a membrane that moisturizes the skin surface more effectively. The DGC membrane suppresses water loss via evaporation from the skin and keeps skin moisturized due to its high water-binding capacity, and could protect skin against irritants in this manner.

Use of a moist, bioactive wound dressing is more likely to promote wound healing than traditional dressings such as gauze; such advanced dressings appear to decrease healing times and the length and risk of infection. The hydrogel system based on poly(ethylene glycol)/protein hydrophilic polymer conjugates provides a favourable environment including interleukin (IL)-1b, IL-6, and transforming growth factor (TGF)-b1 for keratinocyte proliferation. ${ }^{43}$ Based on the findings of the present study, DGC appears to be a promising ingredient for wound dressings.

Table 6 Reduction of erythema response to methyl nicotinate $(\mathrm{MN})$ or hexyl nicotinate $(\mathrm{HN})$ on dorsal skin of guinea pigs after pretreatment with the test samples $^{\mathrm{a}}$

\begin{tabular}{llllllll}
\hline & Groups: sample & I: vehicle & II:WSC & III: DGC & P value $^{\text {d }}$ & & \\
\cline { 2 - 8 } & n & $\mathbf{5}$ & $\mathbf{5}$ & $\mathbf{5}$ & IVs. II & IVs. III & IIVs. III \\
\hline \multirow{2}{*}{ MN-induced erythema } & Mean & 2 & 2 & 0.8 & I & $0.047^{*}$ & $0.047^{*}$ \\
& SD & 0 & 0 & 0.7 & & & \\
HN-induced erythema & Mean & 1.8 & 1.6 & 0.6 & & & \\
& SD & 0.4 & 0.5 & 0.5 & 0.79 & $0.037^{*}$ & 0.079 \\
\hline
\end{tabular}

aEach observed effect was classified as follows: 2 , marked erythema, I, slight or very weak erythema, 0 , no reaction; b Water-soluble chitin; c Dodecylglycylchitin; d Tested by Steel-Dwass test, *, P<0.05

Although the molecular weight of DGC $\left(\mathrm{M}_{\mathrm{n}}=1.5 \times 10^{5}\right)$ is too large to be absorbed via the skin, it is possible that the cells making up the disrupted skin barrier are influenced by chitin and its derivatives. Many studies have shown that chitin accelerated wound healing. Chitin and chitosan as antimicrobial agents, moisturizers, film-forming agents, and bioadhesive agents have been reported to enhance the function of inflammatory cells including neutrophils, macrophages, and fibroblasts. ${ }^{44,45}$ Chitin and its derivatives stimulated the production of IL-8 from rat dermal fibroblasts in vitro. ${ }^{46}$ IL-8 is a major mediator of the inflammatory response and known as neutrophil chemotactic factor, which attracts neutrophils to sites of inflammation. Recently, chitin was also found to stimulate keratinocytes in vitro. ${ }^{47}$ By upregulating Toll-like receptor-4 of keratinocytes, chitin may enhance neutrophilic innate immunity and host defence in bacterial skin infection, especially Gram-negative bacteria such as Pseudomonas aeruginosa. The application of chitosan strongly inhibited itch-related scratching in hairless mice associated with barrier-disrupted dry skin. ${ }^{48}$ The inhibitory mechanism could be explained by recovery of skin barrier function.

Epidermal Langerhans cells (LCs) are dendritic cells (DCs: antigen-presenting immune cells) in the skin. Activated LCs elongate their dendrites to penetrate keratinocyte (KC) tight junctions (TJs), and LCs uptake external antigens that have disrupted the stratum corneum (SC) barrier in the presence of intact TJ barrier function. ${ }^{49}$ Actually, it has become apparent recently that the number of patients with wheat-dependent exercise-induced anaphylaxis (WDEIA) developed after the use of soap that contains wheat protein has been increasing in Japan. ${ }^{50,51}$ Thus, percutaneous sensitization will be an initial trigger of atopic dermatitis, followed by epidermal barrier dysfunction. Most true fungi including microorganisms such as yeasts, moulds, and mushrooms have a cell wall consisting of chitin. We have thought that the topical application of chitin/chitosan and their derivatives to the skin will produce two sets of barriers: physical strength and immunological activity against bacterial infection. Since various peptide epitopes are easily introduced into chitin and chitosan polymers, our experimental method to prepare chitin derivatives using the water-soluble active ester will be useful in the molecular design of percutaneous vaccination.

Chitin/chitosan is an excellent material for the hydrogel system because it has two functions: a wrapping effect as water-soluble biopolymer and a physiological effect via immunostimulatory activity. We propose DGC, a chitin derivative having a lipid/peptide/ chitin structure, as a new class of polymer for use in pharmaceutical preparations for medical skin care such as hydrogels, hydrosheets, or barrier creams.

\section{Conclusion}

The present work demonstrates that the DGC membrane has the ability to restore the function of a disrupted skin barrier. DGC was observed to immediately improve skin barrier function disrupted by n-hexadecane. In addition, DGC suppressed the penetration of various chemical substances into the skin. We propose that DGC is new class 
of polymer made of chitin for use in medical biomaterials that is effective both as preventive and therapeutic skincare.

\section{Acknowledgements}

The authors thank Emeritus Professor Masahiko Okada from Nagoya University who provided valuable suggestions. The authors received generous support from Mr. Hidenobu Okumura, NOEVIR Co, Ltd.

\section{Conflict of interest}

Author declares that there is no conflict of interest.

\section{References}

1. Muzzarelli RA. Chitin. Oxford, UK: Pergamon Press; 1977.

2. Kurita K. Desk Reference of Functional Polymers: Syntheses and applications. In: Arshady R, editor. American Chemical Society, USA; 1977. p. 239-260.

3. Hirano S. Chitin and Chitosan as novel biotechnological materials. Polym Int. 1999;48(8):732-734.

4. Muzzarelli RA, Guerrieri M, Goteri G, et al. The biocompatibility of dibutyryl chitin in the context of wound dressings. Biomaterials. 2005;26(29):5844-5854

5. Murakami K, Ishihara M, Aoki H, et al. Enhanced healing of mitomycin $\mathrm{C}$-treated healing-impaired wounds in rats with hydrosheets composed of chitin/chitosan, fucoidan, and alginate as wound dressings. Wound Repair Regen. 2010;18(5):478-485.

6. Kifune K. Advances in chitin and chitosan. In: Brine CJ, Zikakis JP, editors. UK: Elsevier London; 1992. p. 9-15.

7. Burkatovskaya M, Castano AP, Demidova-Rice TN, et al. Effect of chitosan acetate bandage on wound healing in infected and noninfected wounds in mice. Wound Repair Regen. 2008;16(3):425-431.

8. Kordestani S, Shahrezaee M, Tahmasebi MN, et al. A randomized controlled trial on the effectiveness of an advanced wound dressing used in Iran. J Wound Care. 2008;17(7):323-327.

9. Fujie T, Okamura Y, Takeoka S. Ubiquitous transference of a freestanding polysaccharide nanosheet with the development of a nanoadhesive plaster. Advanced Materials. 2007;19(21):3549-3553.

10. Kofuji K, Akamine H, Qian CJ, et al. Therapeutic efficacy of sustained drug release from chitosan gel on local inflammation. Int J Pharm. 2004;272(1-2):65-78

11. Sinha VR, Singla AK, Wadhawan S, et al. Chitosan microspheres as a potential carrier for drugs. Int J Pharm. 2004;274(1-2):1-33.

12. He W, Guo X, Xiao L, et al. Study on the mechanisms of chitosan and its derivatives used as transdermal penetration enhancers. Int J Pharm. 2009;382(1-2):234-243.

13. Muzzarelli RA. Chitins and chitosans as immunoadjuvants and nonallergenic drug carriers. Mar Drugs. 2010;8(2):292-312.

14. Zhao L, Zhu L, Liu F, et al. pH triggered injectable amphiphilic hydrogel containing doxorubicin and paclitaxel. Int $J$ Pharm. 2011;410(1-2):83-91.

15. Arayachukeat S, Wanichwecharungruang SP, Tree-Udom T. Retinyl acetate-loaded nanoparticles: dermal penetration and release of the retinyl acetate. Int J Pharm. 2011;404(1-2):281-288.

16. Kurita K, Shimada K, Nishiyama Y, et al. Nonnatural Branched Polysaccharides: Synthesis and Properties of Chitin and Chitosan Having alpha-Mannoside Branches. Macromolecules. 1998;31(15):4764-4769.
17. Nishimura SI, Kai H, Shinada K, et al. Regioselective syntheses of sulfated polysaccharides: specific anti-HIV-1 activity of novel chitin sulfates. Carbohydr Res. 1998;306(3):427-433.

18. Jayakumar R, Nwe N, Tokura S, et al. Sulfated chitin and chitosan as novel biomaterials. Int J Biol Macromol. 2007;40(3):175-181.

19. Sannan T, Kurita K, Iwakura Y. Solubility change by alkaline treatment and film casting. Makromol Chem. 1975;176(4):1191-1195.

20. Cho YW, Jang J, Park CR, et al. Preparation and solubility in acid and water of partially deacetylated chitins. Biomacromolecules. 2000;1(4):609-614.

21. Cho YW, Cho YN, Chung SH, et al. Water-soluble chitin as a wound healing accelerator. Biomaterials. 1999;20(22):2139-2145.

22. Aoi K, Takasu A, Okada M. Synthesis of novel chitin derivatives having poly(2-alkyl-2-oxazoline) side chains. Macromolecular Chemistry and Physics. 1994;195(12):3835-3844.

23. Aoi K, Takasu A, Okada M. New chitin-based polymer hybrids. 2. Improved miscibility of chitin derivatives having monodispersepoly (2methyl-2-oxazoline) side chains with poly(vinyl chloride) and poly(vinyl alcohol). Macromolecules. 1997;30(20):6134-6138.

24. Sato H, Tsuge S, Ohtani H, et al. Characterization of Chitin-Based Polymer Hybrids by Temperature-Programmed Analytical Pyrolysis Techniques. 1. Chitin-graft-poly(2-methyl-2-oxazoline)/Poly(vinyl chloride) Blends. Macromolecules. 1997;30(14):4030-4037.

25. Aoi K, Takasu A, Tsuchiya M, et al. New chitin-based polymer hybrids. 3. Miscibility of chitin-graft-poly(2-ethyl-2-oxazoline) with poly(vinyl alcohol). Macromolecular Chemistry and Physics. 1998;199(12):2805-2811.

26. Takasu A, Aoi K, Tsuchiya M, et al. New Chitin-Based Polymer Hybrids, 4: Soil Burial Degradation Behavior of Poly(vinyl alcohol)/ Chitin Derivative Miscible Blends. Journal of Applied Polymer Science. 1999;73(7):1171-1179.

27. Aoi K, Takasu A, Okada M, et al. Synthesis and assembly of novel chitin derivatives having amphiphilic polyoxazoline block copolymer as a side chain. Macromolecular Chemistry and Physics. 1999;200(5):1112-1120.

28. Detchprohm S, Aoi K, Okada M. Synthesis of a Novel Chitin Derivative Having Oligo( $\varepsilon$-caprolactone) Side Chains in Aqueous Reaction Media. Macromolecular Chemistry and Physics. 2001;202(18):3560-3570.

29. Aoi K, Takasu A, Okada M, et al. Nano-Scale Molecular Shapes of Water-Soluble Chitin Derivatives Having Monodisperse Poly(2-alkyl2-oxazoline) Side Chains. Macromolecular Chemistry and Physics. 2002;203(18):2650-2657.

30. Nakamura R, Aoi K, Okada M. Interactions of Enzymes and a lectin with a Chitin-Based Graft Copolymer Having Polysarcosine Side Chains. Macromol Biosci. 2004;4(6):610-615.

31. Seki T, Aoi K, Okada M, et al. Synthesis of chitin derivatives having peptide side groups by the water-soluble active ester method. Macromolecular Chemistry and Physics. 2000;201(4):439-446.

32. O'Regan GM, Sandilands A, McLean WH, et al. Filaggrin in atopic dermatitis. J Allergy Clin Immunol. 2008;122(4):689-693.

33. Osawa R, Akiyama M, Shimizu H. Filaggrin gene defects and the risk of developing allergic disorders. Allergol Int. 2011;60(1):1-9.

34. Takahashi K, Morimoto S, Nakamura H, et al. Improvement of pharmaceutical potential of all-trans retinoic acid with hydroxypropyl$\beta$-cyclodextrin. Journal of Inclusion Phenomena and Macrocyclic Chemistry. 2011;70(3-4):389-396.

35. Ooe M, Seki T, Miura T, et al. Comparative evaluation of wrinkle treatments. Aesthetic Plast Surg. 2013;37(2):424-433. 
36. Rogiers V, EEMCO Group. EEMCO guidance for the assessment of transepidermal water loss in cosmetic sciences. Skin Pharmacol Appl Skin Physiol. 2001;14(2):117-128

37. Aoi K, Seki T, Okada M, et al. Synthesis of a novel $N$-selective ester functionalized chitin derivative and water-soluble carboxyethylchitin. Macromolecular Chemistry and Physics. 2000;201(14):1701-1708.

38. Arashima M, Okumura H, Terai M, et al. Relationship between the pain sensitivity and TEWL on the human skin, in JSID Abstracts. J Dermatol Sci. 1996;12(2):216-216(1).

39. Moloney SJ, Teal JJ. Alkane-induced edema formation and cutaneous barrier dysfunction. Arch Dermatol Res. 1988;280(6):375-379.

40. Tanojo H, Boelsma E, Junginger HE, et al. In vivo human skin barrier modulation by topical application of fatty acids. Skin Pharmacol Appl Skin Physiol. 1998;11(2):87-97.

41. Reinberg AE, Soudant E, Koulbanis C, et al. Circadian dosing time dependency in the forearm skin penetration of methyl and hexyl nicotinate. Life Sci. 1995;57(16):1507-1513.

42. Frosch PJ, Schulze-Dirks A, Hoffmann M, et al. Efficacy of skin barrier creams (I). The repetitive irritation test (RIT) in the guinea pig. Contact Dermatitis. 1993;28(2):94-100.

43. Shingel KI, Di Stabile L, Marty JP, et al. Inflammatory inert poly(ethylene glycol)-protein wound dressing improves healing responses in partialand full-thickness wounds. Int Wound J. 2006;3(4):332-342.
44. Tokura S, Tamura H, Azuma I. Immunological aspects of chitin and chitin derivatives administered to animals. EXS. 1999;87:279-292.

45. Ueno H, Mori T, Fujinaga T. Topical formulations and wound healing applications of chitosan. Adv Drug Deliv Rev. 2001;52(2):105-115.

46. Mori T, Okumura M, Matsuura M, et al. Effects of chitin and its derivatives on the proliferation and cytokine production of fibroblasts in vitro. Biomaterials. 1997;18(13):947-951.

47. Koller B, Müller-Wiefel AS, Rupec R, et al. Chitin modulates innate immune responses of keratinocytes. PLoS One. 2011;6(2):e16594.

48. Fujii M, Shimizu T, Nakamura T, et al. Inhibitory effect of chitosancontaining lotion on scratching response of hairless mice with atopic dermatitis-like dry skin. Biol Pharm Bull. 2011;34(12):1890-1894.

49. Kubo A, Nagao K, Yokouchi M, et al. External antigen uptake by Langerhans cells with reorganization of epidermal tight junction barriers. J Exp Med. 2009;206(13):2937-2946.

50. Matsuo H, Morita E, Tatham AS, et al. Identification of the IgE-binding epitope in omega-5 gliadin, a major allergen in wheat-dependent exerciseinduced anaphylaxis. J Biol Chem. 2004;279(13):12135-12140.

51. Ishii K, Hiragun M, Matsuo H, et al. Remission of Wheat-dependent Exercise-induced Anaphylaxis after the Cessation of Hydrolysed Wheatcontaining Soap Usage. Acta Derm Venereol. 2012;92(5):490-491. 